\title{
Dinâmica espacial da murcha-de-ceratocystis em Khaya senegalensis A. Juss
}

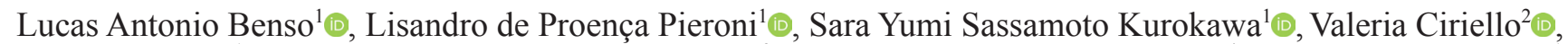
Cristiane de Pieri ${ }^{1} \mathbb{\infty}$, Carlos Alberto Hector Flechtmann ${ }^{3} \mathbb{C}$, Waldir Cintra de Jesus Junior ${ }^{4} \mathbb{C}$, Edson Luiz Furtado ${ }^{1} \mathbb{C}$

${ }^{1}$ Faculdade de Ciências Agronômicas, Universidade Estadual Paulista "Júlio de Mesquita Filho". Departamento de Proteção Vegetal, Av. Universitária, 3780, Altos do Paraíso, CEP: 18610-307, Botucatu-SP, Brasil. ${ }^{2}$ Futuro Florestal, Rua Rodolfo Miranda, 292, Williams, CEP: 17400000, Garça-SP, Brasil. "Faculdade de Engenharia - Universidade Estadual Paulista "Júlio de Mesquita Filho". Departamento de Fitossanidade, Engenharia Rural e Solos. Av. Brasil Sul, 56, Centro, CEP: 15385-000, Ilha Solteira/SP, Brasil. " Universidade Federal de São Carlos, Campus de Lagoa do Sino. Rodovia Lauri Simões de Barros, km 12 - SP-189, Aracaçu, CEP: 18290-000, Buri-SP, Brasil.

Autor para correspondência: Lucas Antonio Benso (benso.florestal@gmail.com)

Data de chegada: 22/04/2019. Aceito para publicação em: 19/01/2021.

$10.1590 / 0100-5405 / 222973$

\section{RESUMO}

Benso, L.A.; Pieroni, L.P.; Kurokawa, S.Y.S.; Ciriello, V.; Pieri, C.; Flechtmann, C.A.H.; Jesus Junior, W.C.; Furtado, E.L. Dinâmica espacial da murcha-de-ceratocystis em Khaya senegalensis A. Juss. Summa Phytopathologica, v.47, n.1, p.38-44, 2021.

O gênero Khaya engloba importantes espécies florestais produtoras de madeira, cujo lenho é utilizado principalmente na indústria moveleira e na construção civil. Dentre os membros desse gênero, Khaya senegalensis é uma das espécies mais cultivadas no Brasil. A murcha-de-ceratocystis está entre as principais doenças dessa cultura, se tornando limitante caso não manejada adequadamente. Até o momento não foram conduzidos estudos epidemiológicos envolvendo essa patologia em $K$. senegalensis. O objetivo deste trabalho foi caracterizar a disseminação da murcha-de-ceratocystis em $K$. senegalensis, por meio da técnica de Análise da Dinâmica e Estrutura de Focos (ADEF) e do Índice de Dispersão (ID). Instalou-se três parcelas com 540 indivíduos cada no interior de um talhão puro dessa espécie florestal. A quantificação da doença foi realizada anualmente de 2016 a 2018, sendo elaborados mapas de dispersão das plantas doentes, os quais serviram de base para as análises. Pela ADEF, observou-se o padrão agregado em todas as parcelas e anos avaliados, com focos apresentando crescimento isodiamétrico. No entanto, pela técnica do ID, o padrão variou entre ao acaso e agregado, em função do tamanho do quadrat utilizado na análise. Pelo tamanho dos focos observados na área experimental, recomendou-se o uso de quadrats maiores no ID, que indicaram um padrão agregado na maior parte das parcelas. Pôde-se concluir que no patossistema estudado houve tendência a agregação das plantas doentes, com uma provável participação da ferramenta de poda no processo de disseminação e/ou infecção pelo agente causal.

Palavras-chave: mogno africano; epidemiologia; disseminação

\section{ABSTRACT}

Benso, L.A.; Pieroni, L.P.; Kurokawa, S.Y.S.; Ciriello, V.; Pieri, C.; Flechtmann, C.A.H.; Jesus Junior, W.C.; Furtado, E.L. Spatial dynamics of Ceratocystis wilt in Khaya senegalensis A. Juss. Summa Phytopathologica, v.47, n.1, p.38-44, 2021.

The genus Khaya includes important forest species, whose wood is used mainly in the furniture industry and civil construction. Khaya senegalensis is one of the most cultivated species in Brazil. Ceratocystis wilt is one of the main diseases of this culture, offering a serious risk if not properly managed. However, no epidemiological studies have been conducted involving this pathology in $K$. senegalensis. The objective of this work was to characterize the spread of Ceratocystis wilt in K. senegalensis, using the Dynamics and Foci Structure Analysis technique (DFSA) and the Dispersion Index (DI). Three plots with 540 individuals each were installed inside a plantation of this species. The quantification of the disease was done annually from 2016 to 2018, with the elaboration of dispersion maps of diseased plants, which served as the basis for all the analyzes performed. According to DFSA, the aggregate pattern was observed in all plots and years evaluated, with disease foci showing isodiametric growth. However, using the ID technique, the pattern varied between random and aggregate, depending on the size of the quadrat used. Due to the size of the foci observed in the experimental area, the use of larger quadrats in the ID was recommended, which indicated an aggregate pattern in most plots. Thus, it was possible to conclude here was a tendency for the aggregation of diseased plants, with a probable participation of the pruning tool in the process of spreading and/ or infection by the causal agent.

Keywords: African mahogany; epidemiology; dissemination

O gênero Ceratocystis contém importantes espécies de fungos fitopatogênicos, reconhecidos como típicos causadores de murchas em espécies arbóreas $(2,6,9,13,21,24)$. Dentro desse gênero, a espécie Ceratocystis fimbriata Ellis \& Halst. é destaque devido à sua ampla gama de hospedeiros, que abrange espécies agronômicas e florestais, e seu potencial em causar sérios prejuízos econômicos as culturas afetadas $(2,4,20)$. A penetração do patógeno no caule de espécies arbóreas é normalmente dependente de ferimentos, que podem ser resultantes de tratos culturais como podas, ou oriundo do ataque de insetos (1,
$5,7,8,10,16,21,24)$. Uma vez no interior da planta, C. fimbriata coloniza os vasos do xilema, associados ao transporte de água e sais da raiz para a parte aérea, resultando em murcha, seca e morte da planta doente $(5,8,20)$. Devido às alterações físico-químicas dos tecidos do lenho colonizado, pode-se observar o escurecimento das células parenquimáticas na forma de estrias radiais, que saem da medula e se dirigem à casca, sendo esse um sintoma visual característico (típico) de plantas afetadas por C. fimbriata. Também pode ocorrer a formação de brotações adventícias abaixo do ponto de penetração do fungo (5). 
Nas culturas do cacau (Theobroma cacao L.) e da seringueira (Hevea brasiliensis (Willd. ex A. Juss.) Müll. Arg.), uma das formas de dispersão de Ceratocystis spp. é através de ferramentas empregadas nos tratos culturais, como o facão utilizado na poda do cacaueiro e a faca de sangria na seringueira $(1,11,21)$. As estruturas reprodutivas do fungo permanecem aderidas a essas ferramentas após serem utilizadas em tecidos contaminados, atuando a partir desse momento como agente de disseminação do patógeno para plantas sadias. A necessidade de realização de podas em algumas espécies florestais suscetíveis à murcha-de-ceratocystis dificulta o manejo da doença, visto que essa prática pode promover tanto a disseminação do patógeno quanto a abertura de portas de entrada para a penetração do fungo.

O estudo do padrão de dispersão espacial de uma doença fornece dados importantes sobre o seu desenvolvimento no interior do plantio, possibilitando uma visão mais detalhada da estrutura e comportamento do patógeno dentro do patossistema $(3,4,12,14,15,17,18,19,25)$. Esses padrões correspondem basicamente ao arranjo formado pelas plantas doentes em relação às sadias dentro do mesmo patossistema, estando fortemente relacionados aos tipos de mecanismos de dispersão responsáveis pela disseminação do patógeno e sua associação com a planta hospedeira (26). Do ponto de vista prático, a aplicação desses estudos possibilita também a avaliação da eficiência de técnicas de manejo empregadas e a quantificação dos danos ocasionados pela doença.

A murcha-de-ceratocystis foi relatada recentemente na cultura da K. senegalensis (9) e ainda são escassos os estudos associados ao patossistema. O objetivo deste trabalho foi caracterizar a disseminação da murcha-de-ceratocystis em $K$. senegalensis, por meio da técnica de Análise da Dinâmica e Estrutura de Focos (ADEF) e do Índice de Dispersão (ID).

\section{MATERIAL E MÉTODOS}

\section{Descrição do local de estudo}

$\mathrm{O}$ experimento foi conduzido em um talhão comercial composto por 2000 plantas de K. senegalensis, em Garça/SP $\left(22^{\circ} 15.880^{\prime} \mathrm{S}\right.$ $\left.49^{\circ} 40.520^{`} \mathrm{~W}\right)$. As árvores foram plantadas em março de 2013, em espaçamento $2,5 \times 2,5 \mathrm{~m}$, utilizando-se de mudas de origem seminal com idade aproximada de 10 meses produzidas com substrato em tubetes. Em torno do talhão foi feito o plantio de linhas de Corymbia citriodora (Hook.) K.D. Hill \& L.A.S. Johnson e de jequitibá-rosa (Cariniana legalis (Mart.) Kuntze), como quebra-vento. Em 2015 foi realizada a poda dos ramos baixos das plantas, momento a partir do qual se verificou o aparecimento de árvores com sintomas de murcha e seca.

\section{Quantificação da doença}

Foram instaladas três parcelas experimentais dentro do talhão estudado, sendo cada parcela composta por 540 indivíduos $(18$ x 30 plantas), demarcadas a aproximadamente $10 \mathrm{~m}$ da borda do talhão, sendo consideradas também como bordaduras as linhas de C. citriodora e C. legalis.

O número e a localização das plantas doentes e sadias foi determinado através do caminhamento dentro do talhão, registrando esses dados em planilha eletrônica Excel, gerando assim os mapas que serviram como base para a análise do padrão espacial da doença. Foram classificadas como árvores doentes aquelas que apresentavam sintomas típicos da murcha-de-ceratocystis: murcha e seca da parte aérea (Figura 1) e escurecimento do lenho em formato de estrias radiais (5), verificado através de cortes do lenho com uso de facão. Adicionalmente, para confirmação do agente causal da doença em questão, amostras de tecidos apresentando descoloração foram coletadas de algumas plantas doentes e isoladas seguindo-se procedimento padrão com uso de iscas de cenoura (22). A partir dos isolamentos verificou-se que o patógeno presente em tais plantas se assemelhava morfologicamente ao fungo $C$. fimbriata. A formação de brotações adventícias, trincas da casca e liberação de resinas no local de infecção foram observadas em praticamente todas as plantas de $K$. senegalensis doentes, e podem ser considerados também como sintomas característicos da murchade-ceratocystis nessa espécie florestal.

O experimento foi conduzido de 2016 a 2018, tendo sido a avaliação realizada no mês de agosto de cada ano.

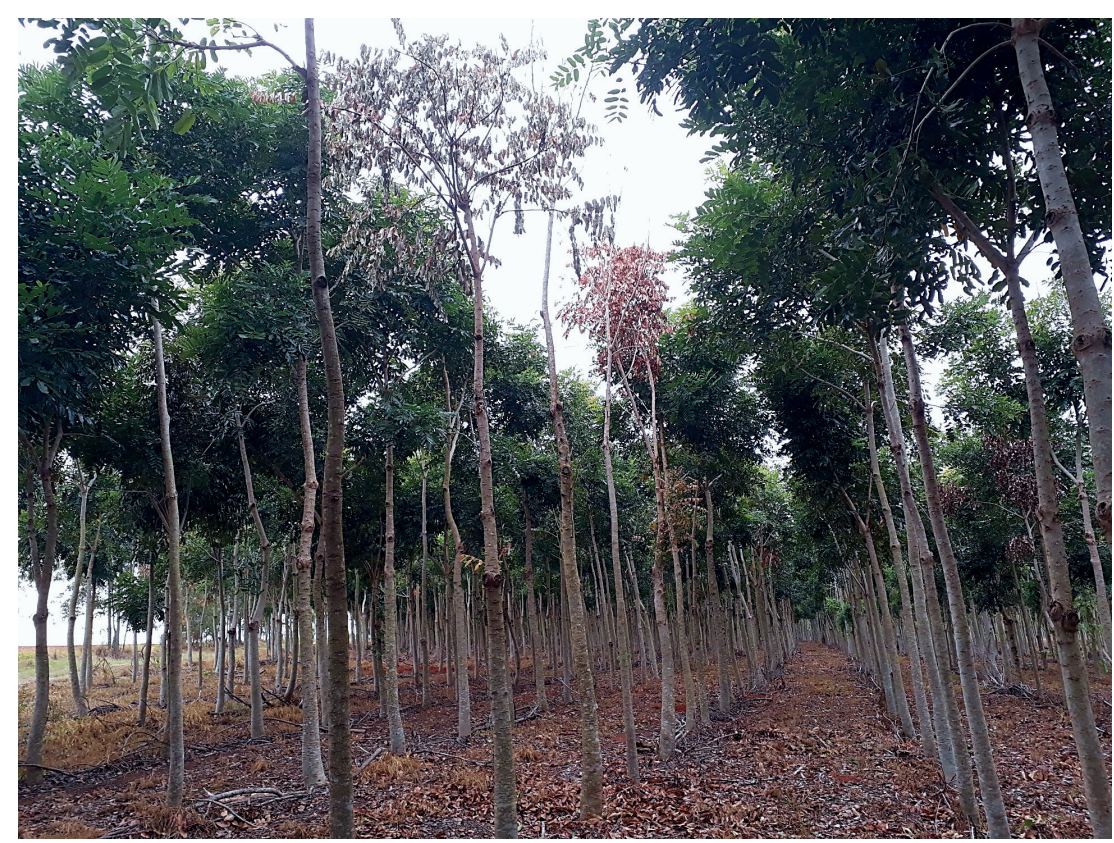

Figura 1. Sintoma de seca causada pela murcha-de-ceratocystis em plantas de mogno africano (Khaya senegalensis). Garça/SP, 2017. 


\section{Análise da Dinâmica e Estrutura dos Focos (ADEF)}

Usando a metodologia empregada por Barbosa (3), Jesus Junior et al. (15) Laranjeira et al. $(17,18)$ e Nelson (23), considerou-se que somente as plantas com sintomas de murcha-de-ceratocystis imediatamente adjacentes no padrão de proximidade vertical, horizontal ou diagonal pertenciam ao mesmo foco. Os focos unitários são aqueles compostos por apenas uma única planta afetada. Em todas as avaliações determinou-se o número de focos (NF), o número de plantas em cada foco i (NPFi) e o número máximo de linhas (lfi) e colunas (lci) ocupadas por cada foco i. A partir desses dados foi calculado o índice médio de forma de focos (IFF, em que IFF $=[\mathrm{S}($ lfi/lci) $] / \mathrm{NF})$ e o índice médio de compactação de focos (ICF, em que ICF $=[\mathrm{S}(\mathrm{NPFi} / \mathrm{lci}$ (lfi) $] / \mathrm{NF})$. Valores de IFF iguais a 1,0 indicam focos isodiamétricos, valores maiores que 1,0 indicam focos com maior comprimento na direção entre as linhas de plantio, e valores menores que 1,0 indicam focos com maior comprimento na direção da linha de plantio. Valores de ICF próximos a 1,0 indicam focos mais compactos, isto é, maior agregação e proximidade entre todas as plantas pertencentes ao foco (23). Todas estas variáveis calculadas foram relacionadas com a incidência de plantas com sintomas de murcha-de-ceratocystis.

\section{Índice de Dispersão (ID)}

O cálculo do ID se baseia no estudo da distribuição das plantas doentes em diferentes tamanhos de quadrats instalados na área amostral $(3,12,18,19)$. Para o cálculo da incidência (p) em cada parcela foi utilizada a equação:

- $\mathrm{p}=\mathrm{D} / \mathrm{T}$

em que:

$\mathrm{T}=$ número total de plantas na parcela;

$\mathrm{D}=$ número total de plantas doentes.

Cada uma das parcelas foi dividida em quadrats de diferentes tamanhos, a saber: $4(2 \times 2), 10(5 \times 2)$ e $36(6 \times 6)$ plantas/quadrat. $\mathrm{O}$ quadrat de tamanho $5 \times 2$ possui maior comprimento na direção da linha de plantio. Para cada mapa e tamanho de quadrats foi feita uma análise individual.

O Índice de Dispersão foi calculado por meio da razão entre a variância observada entre quadrats (Vobs) e a variância da distribuição binominal (Vbin), por meio das equações:

- $\quad \operatorname{Vbin}=\mathrm{p}(1-\mathrm{p}) / \mathrm{n}$

- $\quad$ Vobs $=\Sigma\left[(x i-n p)^{2} / n^{2}(\mathrm{~N}-1)\right]$

- $\quad \mathrm{ID}=\mathrm{Vbin} / \mathrm{Vobs}$

em que:

$\mathrm{p}$ = incidência da doença na parcela;

$\mathrm{n}=$ número de plantas no quadrat;

xi = número de plantas doentes no quadrat;

$\mathrm{N}=$ número total de quadrats.

Valores de ID $<1$ indicam dispersão regular, ID $=1$ indicam dispersão ao acaso e ID > 1, indicam agregação espacial.

Para verificar a significância dos resultados de ID maiores que 1 foi realizado o cálculo do teste do qui-quadrado $\left(\mathrm{X}^{2}\right)$, a $5 \%$ de probabilidade, por meio das equações:

- $\quad \mathrm{X}^{2}$ calculado $=0,5[1,645+(2(\mathrm{~N}-1)-1)]^{2}$

- $\quad \mathrm{X}^{2}$ observado $=$ ID $(\mathrm{N}-1)$

em que:

$\mathrm{N}$ = número total de quadrats.

A hipótese do padrão agregado foi testada através do teste do qui- quadrato. Caso os valores obtidos para $\mathrm{X}^{2}$ observado sejam maiores que o do $\mathrm{X}^{2}$ calculado, aceita-se a hipótese de padrão agregado. Caso contrário, aceita-se o padrão ao acaso $(19,25,26)$.

\section{RESULTADOS E DISCUSSÃO}

Ao longo das avaliações, foi observada a ocorrência de plantas doentes nas três parcelas estudadas, com aumento da incidência da murcha-de-ceratocystis ao longo do tempo (Figura 2). Foi possível verificar uma possível associação entre a doença e as podas realizadas no talhão em 2015, visto que o escurecimento do lenho, a ocorrência de trincas na casca, formação de brotações adventícias e a morte dos tecidos da casca coincidiam com o local dos ferimentos abertos durante a poda. Fato semelhante foi observado nas culturas do kiwi (Actinidia deliciosa (A. Chev.) C. F. Liang \& A. R. Ferguson) e do cacaueiro $(21,24)$, em que o processo de colonização do lenho por Ceratocystis spp. inicia-se no local em que foram realizados ferimentos de poda, estendendo-se ao longo do caule. A penetração do patógeno através do sistema radicular nas plantas de mogno africano no talhão estudado provavelmente foi insignificante, visto que a descoloração do lenho concentrou-se única e exclusivamente nos terços médio e superior do caule das plantas doentes, locais onde são removidos os galhos indesejáveis através do processo de poda.

A incidência máxima da murcha-de-ceratocystis no patossistema estudado variou de $11,2 \%$ (parcela 1 ) a $13,9 \%$ (parcela 3 ) no terceiro ano de avaliação (Figura 2). O maior incremento $(2,5 \%)$ foi observado na parcela 1 , cujos valores de incidência aumentaram de $8,7 \%$ para $11,2 \%$ no período de 2016 a 2018. Ao se analisar a incidência da doença entre os anos, em todas as parcelas, verifica-se maior incremento no período de 2016 a 2017 do que de 2017 para 2018. Esse padrão de evolução da doença, com redução gradativa no aparecimento de novo indivíduos sintomáticos ao longo do tempo, foi observado também por Ferreira et al. (8) no patossistema $C$. fimbriata x eucalipto. Nessa cultura florestal, cuja principal via de penetração do patógeno é através do sistema radicular, a maior manifestação de sintomas externos de murcha e seca ocorre até 20 meses após o plantio em locais com presença abundante de inóculo no solo (8). Nessa fase inicial do desenvolvimento, as plantas são menos tolerantes ao comprometimento da translocação de seiva pelo patógeno, ocorrendo a redução gradativa da mortalidade após essa idade até o momento da colheita. No talhão estudado, a manifestação dos sintomas externos iniciou-se somente no ano posterior à realização das podas, quando as plantas tinham 3,5 anos. Estes resultados indicam que o processo de poda possibilitou a entrada do patógeno nas plantas em 2015, levando à manifestação dos sintomas externos em 2016, com aumentos decrescentes na manifestação dos sintomas nos anos posteriores.

\section{Análise da Dinâmica e Estrutura dos Focos (ADEF)}

Com base na ADEF verificou-se que a murcha-de-ceratocystis apresentou padrão de dispersão do tipo agregado e focos isodiamétricos com leve tendência de dispersão entre as linhas de plantio em todas as avaliações (Tabela 1). As parcelas 2 e 3 apresentaram aumento da compactação dos focos (ICF) no final dos três anos de avaliação, provavelmente ligado à maior proximidade das plantas doentes com o aumento da incidência. Nessas parcelas também foi verificada a redução no número de focos unitários (NFU) de 2016 para 2018, com consequente redução no número total de focos (NF) e aumento no número médio de plantas por foco (NMPF). A parcela 1 apresentou 


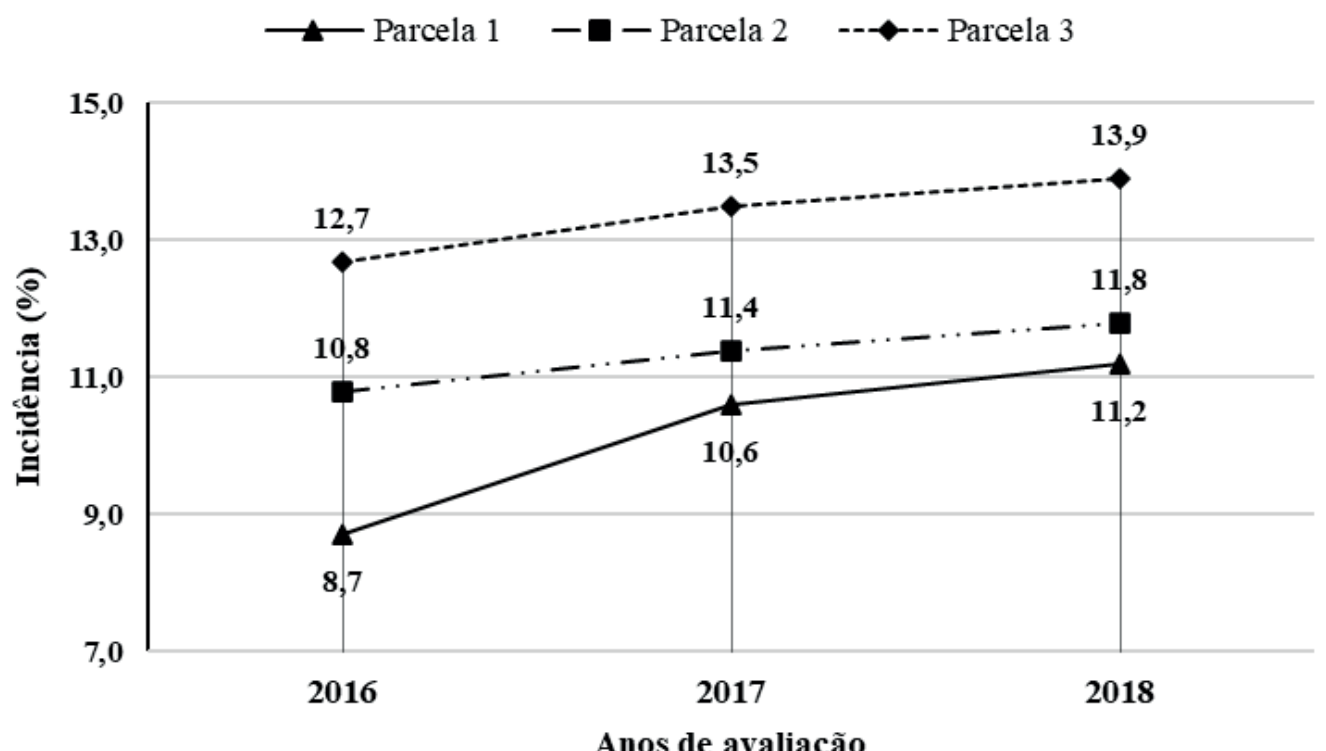

Figura 2. Flutuação da incidência (\%) da murcha-de-ceratocystis em três parcelas experimentais de Khaya senegalensis em plantio comercial situado no município de Garça/SP, entre 2016 e 2018.

Tabela 1. Incidência (\%) da murcha-de-ceratocystis, número de plantas doentes (NPD), número total de focos (NF), número de focos unitários (NFU), número médio de plantas por foco (NMPF), índice médio de forma de focos (IFF) e índice médio de compactação de focos (ICF) em três parcelas experimentais de Khaya senegalensis avaliadas em plantio comercial situado no município de Garça/SP, entre 2016 e 2018.

Parcela 1

\begin{tabular}{cccccccc}
\hline Avaliações & Incidência & NPD & NF & NFU & NMPF & IFF & ICF \\
\hline 2016 & 8,7 & 43 & 26 & 18 & 1,65 & 1,2 & 2,1 \\
2017 & 10,6 & 56 & 37 & 26 & 1,51 & 0,99 \\
2018 & 11,2 & 59 & 37 & 26 & 1,59 & 1,16 & 2,1 \\
\hline
\end{tabular}

Parcela 2

\begin{tabular}{cccccccc}
\hline Avaliações & Incidência & NPD & NF & NFU & NMPF & IFF & ICF \\
\hline 2016 & 10,8 & 53 & 41 & 37 & 1,29 & 1,05 & 1,43 \\
2017 & 11,3 & 56 & 40 & 34 & 1,40 & 1,06 \\
2018 & 11,7 & 58 & 40 & 33 & 1,45 & 1,06 & 1,63 \\
\hline
\end{tabular}

Parcela 3

\begin{tabular}{ccccccccc}
\hline Avaliações & Incidência & NPD & NF & NFU & NMPF & IFF & ICF \\
\hline 2016 & 12,7 & 66 & 44 & 34 & 1,50 & 1,1 & 1,67 \\
2017 & 13,6 & 70 & 41 & 30 & 1,71 & 1,08 \\
2018 & 13,9 & 72 & 41 & 30 & 1,76 & 1,11 & 1,85 \\
\hline
\end{tabular}


comportamento distinto, com aumento do número de focos unitários em 2017, levando a uma queda na compactação dos focos. Contudo, com o aumento da incidência em 2018, o valor do ICF atingiu o mesmo nível de 2016. Tal fato provavelmente está relacionado à aleatoriedade temporal do surgimento de planta sintomáticas, sendo esse processo mais marcante no ano de 2016-2017 na parcela 1. Nessa parcela também foram verificados os maiores valores de compactação do foco (ICF) em 2016 e 2018, o que pode ser explicado em função do número relativamente baixo de focos unitários ao longo das avaliações.

O padrão de dispersão do tipo agregado aqui observado já foi constatado no patossistema $C$. fimbriata x eucalipto, sendo que nessa cultura os focos de plantas doentes apresentam crescimento na direção da linha do plantio $(8,26)$. Porém, diferentemente do que verificado no presente estudo, na cultura do eucalipto a distribuição do inóculo no solo provavelmente é o principal fator associado a dispersão do patógeno e, consequentemente, das plantas doentes.

Nas parcelas estudadas, a disseminação do patógeno pode estar relacionado a diferentes mecanismos de disseminação. Em um dos possíveis mecanismos, que envolve o processo de poda das plantas, a ocorrência e desenvolvimento da doença pode ser favorecida de duas maneiras: [1] a ferramenta de poda pode tanto atuar na disseminação do patógeno quanto na abertura de portas de entrada para penetração do fungo pelos ferimentos produzidos; ou [2] somente atuar abrindo ferimentos para penetração do patógeno. Pelo fato do crescimento dos focos apresentar um padrão isodiamétrico de disseminação das plantas doentes, a hipótese mais plausível passa a ser a [2], dado que se a poda fosse o único mecanismo envolvido na disseminação do patógeno teria sido detectado um crescimento dos focos principalmente na direção das linhas/ruas de plantio, o que não aconteceu. O padrão isodiamétrico verificado possibilita que outras hipóteses sejam levantadas sobre os mecanismos de disseminação. Em outras espécies florestais, a disseminação de patógenos fúngicos causadores de murchas pode estar relacionado a outros agentes de disseminação, como por exemplo insetos. Em áreas naturais de Quercus spp., besouros da família Nitidulidae adquirem estruturas reprodutivas de Bretziella fagacearum (T. W. Bretz) J. Hunt, fungo causador da murcha do carvalho, ao caminhar sobre tecidos com esporulação do patógeno em sua superfície e as transportam até o hospedeiro sadio $(10,16)$. Esses besouros são tipicamente atraídos pelas gomas exsudadas de tecidos vegetais feridos, que exalam aroma fermentado, como no caso dos ferimentos oriundos de podas, realizando a inoculação do patógeno nesses tecidos predispostos a infecção $(10,16)$. Em relação ao patossistema estudado ainda são necessários mais estudos para comprovar se existe uma associação entre insetos e a disseminação da murcha-de-ceratocystis.

Tabela 2. Incidência (\%) da murcha-de-ceratocystis, índice de dispersão (ID), $X^{2}$ calculado e $X^{2}$ observado, em três parcelas experimentais de Khaya senegalensis avaliadas em plantio comercial situado no município de Garça/SP, entre 2016 e 2018, para 3 tamanhos de quadrats (6x6 plantas, $5 \times 2$ plantas e $2 \times 2$ plantas).

\section{Parcela 1}

\begin{tabular}{|c|c|c|c|c|c|c|c|c|c|c|}
\hline \multirow{2}{*}{ Avaliações } & \multirow{2}{*}{ Incidência } & \multicolumn{3}{|c|}{$6 \times 6$} & \multicolumn{3}{|c|}{$5 \times 2$} & \multicolumn{3}{|c|}{$2 \times 2$} \\
\hline & & ID & $\mathrm{X}^{2}$ calc & $\mathrm{X}^{2} \mathrm{obs}$ & ID & $\mathrm{X}^{2}$ calc & $\mathrm{X}^{2}$ obs & ID & $\mathrm{X}^{2}$ calc & $\mathrm{X}^{2} \mathrm{obs}$ \\
\hline 2016 & 8,7 & 1,9 & 161,73 & 256,35 & 1,1 & 70,71 & 59,46 & 1,1 & 23,40 & 15,17 \\
\hline 2017 & 10,6 & 1,5 & 161,73 & 203,31 & 1,4 & 70,71 & 73,76 & 1,2 & 23,40 & 16,19 \\
\hline 2018 & 11,2 & 1,8 & 161,73 & 244,07 & 1,3 & 70,71 & 70,67 & 1,1 & 23,40 & 15,92 \\
\hline
\end{tabular}

\section{Parcela 2}

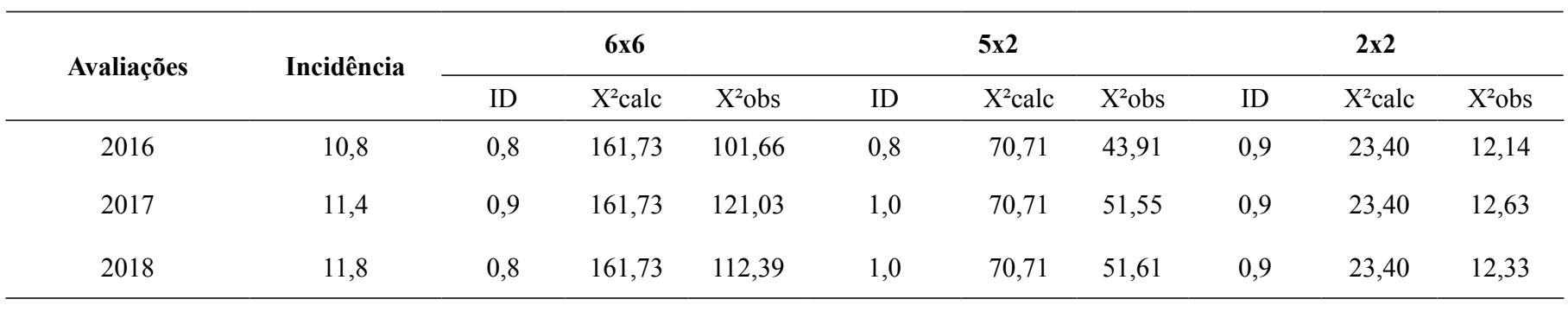

Parcela 3

\begin{tabular}{ccccccccccc}
\hline \multirow{2}{*}{ Avaliações } & \multirow{2}{*}{ Incidência } & \multicolumn{3}{c}{$\mathbf{6 x 6}$} & \multicolumn{3}{c}{$\mathbf{5 x 2}$} & \multicolumn{2}{c}{$\mathbf{2 \times 2}$} \\
\cline { 3 - 10 } & & ID & $\mathrm{X}^{2}$ calc & $\mathrm{X}^{2}$ obs & ID & $\mathrm{X}^{2}$ calc & $\mathrm{X}^{2}$ obs & ID & $\mathrm{X}^{2}$ calc & $\mathrm{X}^{2}$ obs \\
\hline 2016 & 12,7 & 2,3 & 161,73 & 301,50 & 1,0 & 70,71 & 53,91 & 1,0 & 23,40 & 13,56 \\
2017 & 13,5 & 2,1 & 161,73 & 276,12 & 1,0 & 70,71 & 53,43 & 1,0 & 23,40 & 13,91 \\
2018 & 13,9 & 1,9 & 161,73 & 252,69 & 1,0 & 70,71 & 54,27 & 1,0 & 23,40 & 13,94 \\
\hline
\end{tabular}




\section{Índice de dispersão (ID)}

No patossistema estudado, a murcha-de-ceratocystis apresentou padrão de dispersão agregado em $26 \%$ das avaliações nos três tamanhos de quadrats, sendo esse resultado mais frequente no maior tamanho de quadrat (6x6) (Tabela 2). O menor tamanho de quadrat não apresentou nenhum resultado agregado, independendo da parcela ou ano avaliado. A parcela 2 não apresentou em nenhum ano ou tamanho de quadrat o padrão agregado de disseminação das plantas doentes, estando esse resultado provavelmente relacionado ao menor número médio de plantas por foco (NMPF) e maior número de focos unitários (NFU) dessa parcela, quando comparada com as demais (Tabela 2). O impacto do NFU no padrão de dispersão do ID foi discutido também no estudo do ID da clorose variegada (CVC) na cultura da laranja pera (Citrus sinensis (L.) Osbeck) (18). Nesse trabalho foi observado o padrão agregado em quadrats de tamanho $3 \times 3$ e $3 \times 5$, indicando que os focos presentes no local do estudo possuem de 9 a 15 plantas, coincidindo com o resultado encontrado por Barbosa (3), com número médio de 11 plantas apresentando sintomas de CVC em cada foco.

A parcela 3 apresentou os maiores valores do ID (em torno de 2,0) em todos os anos quando empregados quadrats com dimensão 6x6, indicando um forte padrão agregado de dispersão das plantas doentes. No entanto, ao utilizar quadrats de $5 \times 2$ plantas, o padrão observado foi o ao acaso, indicando que os focos, devido ao seu crescimento isodiamétrico, tendem a apresentar padrão ao acaso de dispersão em quadrats com maior comprimento na direção da rua de plantio. Comportamento semelhante foi observado na parcela 2, contudo com menor variação nos valores de ID entre o tamanho de quadrat 6x6 e $5 \times 2$ (Tabela 2).

Normalmente quadrats maiores tendem a englobar um maior número de plantas doentes, sendo mais apropriados no estudo de patossistemas que formam focos maiores, evitando assim o desvio a uma tendência ao acaso. Já os menores são mais sensíveis a doenças ainda em baixa incidência, reduzindo os desvios no índice provocados pela presença de focos unitários dentro dos quadrats. Contudo, no caso dos quadrats menores, focos grandes podem ser interpretados como separados e áreas adjacentes podem acabar por interferir nos resultados. Essa questão foi levantada também por Souza (25), que recomendou o uso de unidades que englobassem um número maior de plantas doentes para estudar focos maiores e evitar desvios. No patossistema estudado foram observados focos com dimensões que chegaram até 6 plantas na direção da linha ou entre as linhas de plantio, sendo recomendado o uso de quadrats maiores, como o tamanho 6x6 empregado nesse trabalho, visando reduzir a interferência do número de focos unitário (NFU) em relação aos focos compostos por mais de uma planta. Quadrats maiores indicaram uma tendência ao padrão agregado de dispersão no patossistema estudado.

\section{Panorama geral do patossistema}

Com base nos resultados obtidos, concluímos que a disseminação da murcha-de-ceratocystis, na condição do plantio comercial de $K$. senegalensis estudado, apresentou tendência a agregação das plantas doentes. O padrão isodiamétrico detectado possibilita que algumas hipóteses sejam levantadas sobre os mecanismos de disseminação do patógeno na área. Provavelmente há mais de um mecanismo envolvido na disseminação do agente causal da doença, sendo que a ferramenta utilizada na poda das plantas pode tanto atuar na disseminação do patógeno quanto na abertura de portas de entrada para penetração do fungo pelos ferimentos produzidos, sendo mais plausível a hipótese que a ferramenta age somente abrindo a porta de entrada. Outra possível forma de disseminação é através de insetos que possam atuar transportando estruturas reprodutivas do patógeno até os ferimentos de poda, no entanto mais estudos são necessários para verificar essa associação.

\section{AGRADECIMENTOS}

Os autores agradecem à CAPES pela bolsa concedida ao primeiro autor e à empresa Futuro Florestal pelo auxílio em todas as etapas de desenvolvimento da pesquisa.

\section{REFERÊNCIAS}

1. AlbuQUerQue, F.C.; DUARTE, M.L.O.; SILVA, H.M. Ocorrência do mofo cinzento (Ceratocystis fimbriata) da seringueira. In: Seminário Nacional da Seringueira, 1., 1972, Cuiabá. Anais. Cuiabá: SUDHEVEA, 1972, p.125-128

2. BAKER, C.J.; HARRINGTON, T.C.; KRAUSS, U.; ALFENAS, A.C. Genetic Variability and Host Specialization in the Latin American Clade of Ceratocystis fimbriata. Phytopathology, St. Paul, v. 93, n. 10, p. 12741284, 2003.

3. BARBOSA, F.F.L. Dinâmica espacial e temporal da clorose variegada dos citros. Dissertação de Mestrado). 1997. 144f. Dissertação (Mestrado em Agronomia) - Escola Superior de Agricultura Luiz de Queiroz, Universidade de São Paulo, Piracicaba.

4. FERNANDES, B.V. Dinâmica espaço-temporal e quantificação dos danos e perdas da murcha de Ceratocystis em eucalipto na produção de carvão vegetal. 2015. 77f. Dissertação (Mestrado em Ciência Florestal) - Faculdade de Ciências Agronômicas, Universidade Estadual Paulista, Botucatu.

5. FERREIRA, F.A.; MAFFIA, L.A.; BARRETO, R.W.; DEMUNER, N.L.; PIGATTO, S. Sintomatologia da murcha de Ceratocystis fimbriata em eucalipto. Revista Árvore, Viçosa, v.30, n.2, p.155-162, 2006.

6. FERREIRA, E.M.; HARRINGTON, T.C.; THORPE, D.J.; ALFENAS, A.C. Genetic diversity and interfertility among highly differentiated populations of Ceratocystis fimbriata in Brazil. Plant Pathology, Londres, v.59, n. 4, p.721-735, 2010.

7. FERREIRA, E.M.; HARRINGTON, T.C.; ALFENAS, A.C.; MIZUBUTI, E.S.G. Movement of genotypes of Ceratocystis fimbriata within and among eucalyptus plantations in Brazil. Phytopathology, St. Paul, v. 101, n. 8 p.1005-1012, 2011.

8. FERREIRA, M.A.; HARRINGTON, T.C.; GONGORA-CANUL, C.C.; MAFIA, R.G., ZAUZA, E.A.V.; ALFENAS, A.C. Spatial-temporal patterns of Ceratocystis wilt in Eucalyptus plantations in Brazil. Forest Pathology, Nova York, v.43, n.2, 2013.

9. FIRMINO, A.C.; MATOS, A.M.S.; TOZZE JR, H.J.; BARRETTO, V.C.M. First report of Ceratocystis fimbriata causing wilt on Khaya senegalensis. New Disease Reports, Londres, v. 35, n. 1, p. 35, 2017.

10. GIBBS, J.N.; FRENCH, D.W. The transmission of oak wilt. St. Paul: U.S. Departamento de Agricultura, 1980. 17p.

11. GONÇALVES, R.C.; VALIM, J.H.; SALDANHA, D. Estudo de prevalência de fungos em painel de sangria de seringueira no acre. In: Congresso Brasileiro de Heveicultura, 3., 2013, Guarapari. Anais. Guarapari: Cedagro, 2013, p.1-4.

12. GOTTWALD, T.R.; CAMBRA, M.; MORENO, P.; CAMARASA, E.; PIQUER, J. Spatial and temporal analyses of citrus tristeza virus in eastern Spain. Phytopathology, St. Paul, v.86, n.1, p.45-55, 1996.

13. HARRINGTON, T.C.; THORPE, D.J.; ALFENAS, A.C. Genetic variation and variation in aggressiveness to native and exotic hosts among Brazilian populations of Ceratocystis fimbriata. Phytopathology, St. Paul, v.101, n.5, p.555-566, 2011.

14. HUGHES, G.; MADDEN, L.V. Aggregation and incidence of disease. Plant Pathology, Londres, v.41, n.6, p.657-660, 1992.

15. JESUS JUNIOR, W.C.; BASSANEZI, R.B.J. Análise da dinâmica e estrutura de focos da morte súbita dos citros. Fitopatologia Brasileira, Viçosa, v.29, n.4, p.399-405, 2004.

17. JUZWIK, J.; SKALBECK, T.C.; NEUMAN, M.F. Sap Beetle Species (Coleoptera: Nitidulidae) Visiting Fresh Wounds on Healthy Oaks During Spring on Minnesota. Forest Science, Maryland, v.50, n.6, p.757-764, 2004.

17. LARANJEIRA, F.F.; AMORIM, L.; BERGAMIN FILHO, A.; BERGER, R.D.; HAU, B. Análise espacial do amarelecimento fatal do dendezeiro 
como ferramenta para elucidar sua etiologia. Fitopatologia Brasileira, Viçosa, v.23, n.3, p.397-403, 1998.

18. LARANJEIRA, F.F.; BERGAMIN FILHO, A.; AMORIM, L.; GOTTWALD, T.R. Dinâmica espacial da clorose variegada dos citros em três regiões do Estado de São Paulo. Fitopatologia Brasileira, Viçosa, v.29, n.1, p.56-65, 2004.

19. MADDEN L.V.; HUGHES, G. Plant disease incidence: distributions, heterogeneity and temporal analysis. Annual Review of Phytopathology, Palo Alto, v.33, n.1, p.529-564, 1995.

20. MAFIA, R.G.; FERREIRA, M.A.; ZAUZA, E.A.; SILVA, J.F.; COLO€ DETTE, J.L.; ALFERNAS, A.C. Impact of Ceratocystis wilt on eucalyptus tree growth and cellulose pulp yield. Forest Pathology, New York, v.43, n.5, 2013.

21. MALAGUTI, G. Ceratostomella fimbriata en el cacao de Venezuela. Acta Cientifica Venezolana, Caracas, v.3, n.3, p.94-97, 1952.

22. MOLLER, W.J.; DEVAY, J.E. Carrot as a species selective isolation medium for Ceratocystis fimbriata. Phytopathology, St. Paul, v.58, n.1, p.123-124, 1968.
23. NELSON, S.C. A simple analysis of disease foci. Phytopatology, St. Paul, v.86, n.4, p.332-339, 1996.

24. PIVETA, G.; ALFENAS, A.C.; MUNIZ, M.DE F.B.; VALDEBENITO-SANHUEZA, R.M.; FERREIRA, M.A. Ocorrência de Ceratocystis fimbriata em kiwi (Actinidia deliciosa) no sul do Brasil. Revista Brasileira de Fruticultura, Jaboticabal, v.35, n.2, p.665-669, 2013.

25. SOUZA, S.E. Dinâmica espaço-temporal e danos do cancro basal em Eucalyptus grandis. 2007. 160f. Tese (Doutorado em Agronomia/Proteção de Plantas) - Faculdade de Ciências Agronômicas, Universidade Estadual Paulista, Botucatu.

26. TUMURA, K.G.; DE PIERI, C.; FURTADO, E.L. Murcha por Ceratocystis em eucalipto: avaliação de resistência e análise epidemiológica. Summa Phytopathologica, Botucatu, v.38, n.1, p.54-60, 2012.

27. VAN DER PLANK, J. E. Plant diseases: epidemics and control. New York: Academic Press, 1963. 349p. 